\title{
TUMOR OF DISTAL TIBIA - MASQUERADING AS TENOSYNOVITIS IN AN ELDERLY FEMALE - A CASE REPORT
}

\section{Orthopaedics}

\section{Dr Stani Mathew*}

\section{Dr Lawrence Mathias}

Junior Resident, Department of Orthopaedics, K.S.Hedge Medical Academy, Deralakatte, Mangalore *Corresponding Author

Professor and Head, Department of Orthopaedics, K.S.Hedge Medical Academy, Deralakatte, Mangalore

\section{ABSTRACT}

INTRODUCTION : Primary bone lymphoma is a rare disease, accounting for about $3 \%$ of all primary bone malignancies in adults. Patients with primary NHL of bone commonly present with local bone pain, soft tissue swelling, and a mass or a pathological fracture. There is a slight male preponderance, and most patients are over 45-50 years of age. Primary NHL of bone can arise in any part of skeleton, but long bones (femur) is the most common sites of presentation but distal tibia is an unusual,rare site of presentation. The outcome of patients with primary lymphoma of bone is relatively favorable with multidrug regimen chemotherapy $+/$ - radiation therapy

CASE PRESENTATION : A 50 year old female presented with chief complaints of pain and swelling over the left leg since 2 months, which was insidious in onset, gradually progressive in nature and with low grade intermittent fever since 1 month. There was no preceding history of trauma, joint stiffness or other associated constitutional symptoms. She had an episode of enteric fever 2 months ago, which was treated with IV antibiotics. She gives history of chemo-radiation for oral malignancy 17 years back, the details of which were not available to us at presentation. She has also undergone hysterectomy 14 years back, following which she had no complications. On general examination, patient had stable vitals, with no pallor or generalised lymphadenopathy. She had left sided non-pitting pedal edema upto the level of mid-calf.

On examination of the right leg, we found that she had multiple localized swellings noted over the distal third of leg. The skin over the swelling was normal, with no wounds, scars, sinuses or dilated veins over the leg. On palpation, there was local rise of temperature with tenderness over the left leg swelling. The swelling had a smooth surface with ill-define margins, soft in consistency with no evidence of crepitus or abnormal movements. Peripheral pulsations were well palpated, and there was no sensory or motor deficits. On movement of left ankle joint, the terminal movements were painful and restricted.Patient was investigated further, where routine blood investigations and X-rays were done, which were found to be inconclusive.MRI of right leg was done to rule out soft tissue infections or osteomyelitis - which revealed a large infiltrative soft tissue lesion over the ankle and lower one third of right leg encasing the tendons, distal tibia and fibula , muscles, skin and subcutaneous tissue with cortical erosions and marrow edema in distal tibia and fibula. A differential diagnosis of synovial sarcoma or metastasic lesion to distal tibia/fibula was suggested based on the MRI report.In view of features suggestive of metastasis, oncology opinion was sought, where they advised USG abdomen and pelvis \& chest x-ray.USG abdomen and pelvis was suggestive of bilateral metastases to adrenal glands with retroperitoneal lymphadenopathy. To confirm the same, CECT abdomen and pelvis, with thorax screening was ordered. This revealed multiple retroperitoneal enlarged lymp nodes with multiple metastases to adrenal and kidneys. Hence, we considered a possibility of lymphoma based on these finding.For the purposes of staging, bone marrow aspiration and biopsy was done, which was found to be normal. With all preliminary investigation done to diagnose the primary lesion, it was decided to perform a biopsy of the lesion from left distal tibial metaphysis and soft tissue swelling.Histopathology revealed a lympho proliferative lesion suggestive of Non Hodgkin`s Lymphoma.Immunohistochemistry markers were ordered on the histopathology specimen, which showed positivity for CD 45, CD 20 , Ki 67 and was confirmed to have Diffuse Large B cell Lymphoma.Patient was then referred to the Oncology department for chemotherapy. Patient is on regular follow-up since then, and remains asymptomatic.

CONCLUSION: Primary lymphoma of the bone involving distal tibia is a very rare entity, with chemotherapy and radiotherapy being the mainstay of treatment. Surgery has a limited role.Early diagnosis and treatment can help reduce significant morbidity and mortality.

\section{KEYWORDS}

NHL ,CHOP, primary lymphoma

\section{INTRODUCTION}

Primary lymphomas of bone are uncommon malignancies. The vast majority of them are non-Hodgkin lymphoma (NHL), whereas primary Hodgkin lymphoma of bone is extremely rare. Non-Hodgkin's lymphoma belongs to group of hematological malignancies originating from T cell or B cell. Primary bone lymphoma is a rare disease, accounting for about $3 \%$ of all primary bone malignancies in adults. Patients with primary NHL of bone commonly present with local bone pain, soft tissue swelling, and a mass or a pathological fracture. There is a slight male preponderance, and most patients are over 45-50 years of age. Primary NHL of bone can arise in any part of skeleton, but long bones (femur) is the most common sites of presentation but distal tibia is an unusual,rare site of presentation. Comprehensive immunohistochemical studies are required to establish an accurate histological diagnosis of primary NHL of bone. Several studies indicate that patients with primary NHL of bone have a favorable outcome. The outcome of patients with primary lymphoma of bone is relatively favorable with multidrug regimen chemotherapy $+/$ - radiation therapy .Doses of radiation in the range of 30-40 Gy provide optimal local control with an acceptable rate of complications. Here we present a case of an elderly female with an incidentally detected Non Hodgkins Lymphoma of distal tibia. Post chemoradiation, she remains asymptomatic and has not shown any signs of recurrence so far.

Case report-

A 50 year old female presented with chief complaints of pain and swelling over the left leg since 2 months, which was insidious in onset, gradually progressive in nature and with low grade intermittent fever since 1 month. There was no preceding history of trauma, joint stiffness or other associated constitutional symptoms. She had an episode of enteric fever 2 months ago, which was treated with IV antibiotics. She gives history of chemo-radiation for oral malignancy 17 years back, the details of which were not available to us at presentation. She has also undergone hysterectomy 14 years back, following which she had no complications. On general examination, patient had stable vitals, with no pallor or generalised lymphadenopathy. She had left sided non-pitting pedal edema upto the level of mid-calf.

On examination of the right leg, we found that she had multiple localized swellings noted over the distal third of leg. The skin over the swelling was normal, with no wounds, scars, sinuses or dilated veins over the leg. On palpation, there was local rise of temperature with tenderness over the left leg swelling. The swelling had a smooth surface with ill-define margins, soft in consistency with no evidence of crepitus or abnormal movements. Peripheral pulsations were well palpated, and there was no sensory or motor deficits. On movement of left ankle joint, the terminal movements were painful and restricted.(Figure 1)

Patient was investigated further, where routine blood investigations and X-rays were done, which were found to be inconclusive.(Figure 2) 
MRI of right leg was done to rule out soft tissue infections or osteomyelitis - which revealed a large infiltrative soft tissue lesion over the ankle and lower one third of right leg encasing the tendons, distal tibia and fibula, muscles, skin and subcutaneous tissue with cortical erosions and marrow edema in distal tibia and fibula. A differential diagnosis of synovial sarcoma or metastasic lesion to distal tibia/fibula was suggested based on the MRI report.(Figure 3)

In view of features suggestive of metastasis, oncology opinion was sought, where they advised USG abdomen and pelvis \& chest $\mathrm{x}$-ray.

USG abdomen and pelvis was suggestive of bilateral metastases to adrenal glands with retroperitoneal lymphadenopathy. To confirm the same, CECT abdomen and pelvis, with thorax screening was ordered. This revealed multiple retroperitoneal enlarged lymp nodes with multiple metastases to adrenal and kidneys. Hence, we considered a possibility of lymphoma based on these finding(Figure 4)

For the purposes of staging, bone marrow aspiration and biopsy was done, which was found to be normal.

With all the preliminary investigation done to diagnose the primary lesion, it was decided to perform a biopsy of the lesion from left distal tibial metaphysis and soft tissue swelling Histopathology revealed a lympho proliferative lesion suggestive of Non Hodgkin`s Lymphoma (Figure 5)

Immunohistochemistry markers were ordered on the histopathology specimen, which showed positivity for CD 45, CD 20, Ki 67 and was confirmed to have Diffuse Large B cell Lymphoma.

Patient was then referred to the Oncology department for chemotherapy. Patient is on regular follow-up since then, and remains asymptomatic.

\section{DISCUSSION:-}

Non-Hodgkin's lymphoma belongs to a group of hematological malignancies that originate either from $\mathrm{T}$ cell or B cell. These vary significantly in their cell of origin, cytogenetics, morphology and prognosis. Generalised lymphadenopathy remains the most common presentation of NHL, which may or may not be associated with hepatosplenomegaly. Involvement of bone as a primary lesion is relatively rare, accounting for less than $2 \%$ of the lymphomas affecting adults [1]. This is seen more common in paediatric population, seen in $3-9 \%$ of cases of NHL. Men are affected more frequently than women, with a ratio ranging from 1.2 to 1.8 [2-5]. The most common age of presentation is more than 30 years, with over $50 \%$ of patients being over 60 years.

The most common presentation of patients with primary lymphoma of bone is deep seated bone pain, not relieving upon rest. There can be associated symptoms such as fever, loss of weight and night sweats. Occasionally, patient presents with features of pathological fractures following trivial trauma and even features of spinal cord compression.

Diagnosis is confirmed by histopathology, where the tissue sample is obtained via percutaneous or open biopsy. A comprehensive history and physical examination is essential in a case of primary lymphoma of bone as it is required to identify all involved sites and any evidence of constitutional symptoms. Imaging is required to identify the primary as well as to look for other extranodal sites of involvement, which includes CT/MRI of the primary site, along with PET/CT of the thorax and abdomen

Multidrug regimen chemotherapy $+/$ - radiation therapy is the preferred treatment of adults with PLB. Given that the majority of PLB are aggressive B cell lymphoma, patients are typically treated with anthracycline-based, multi-agent chemotherapy, such as CHOP regimen (cyclophosphamide, doxorubicin, vincristine and prednisone) with the addition of the anti-CD20 monoclonal antibody Rituximab [5-7]. In patients with unifocal disease, consolidative involved-field radiotherapy is reasonable, but should be individualized with particular attention to the location of disease. Radiation to areas, such as the pelvis, which contain significant marrow production, should be considered carefully. A dose of 30 to $36 \mathrm{~Gy}$ is reasonable in patients who attain a complete response following chemotherapy. A higher dose (eg, $40 \mathrm{~Gy}$ ) is offered to patients with an indeterminant response [7-10].
The role of surgery is generally limited to diagnostic biopsy and stabilization of a pathologic fracture. Orthopedic management is important during the treatment and recovery period because the potential for fracture persists until there is complete bone healing. Rarely, patients with involvement of the weight-bearing bones may require internal stabilization or bracing until bone healing occurs.

\section{CONCLUSION}

Primary lymphoma of the bone involving distal tibia is a very rare entity, with chemotherapy and radiotherapy being the mainstay of treatment. Surgery has a limited role.Early diagnosis and treatment can help reduce significant morbidity and mortality.

\section{Clinical Message:}

Non Hodgkin`s Lymphoma involving the distal tibia is a rare entity with diffuse large $\mathrm{B}$ cell lymphoma being the most commonest type. Chemoradiation is regarded as the main stay of treatment with regular follow up

\section{FIGURES-}

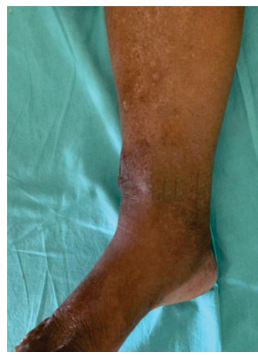

Figure 1 : Initial clinical presentation of swelling over right distal third of leg extending to ankle

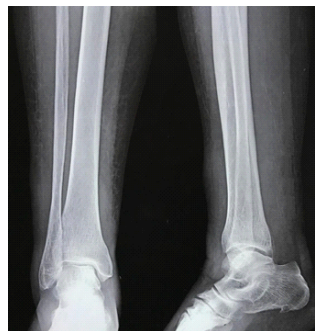

Figure 2: Radiograph of right leg with ankle appears normal

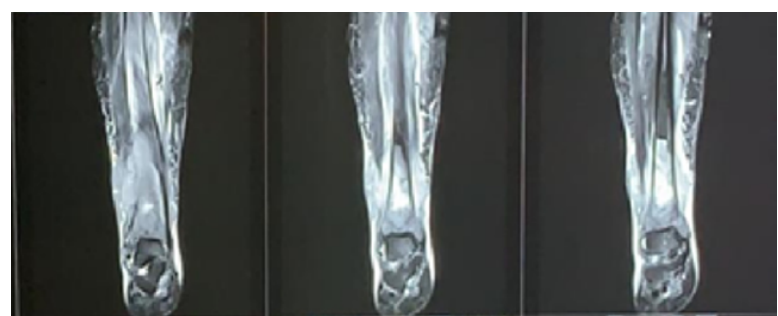

Figure 3: MRI showing large infiltrative soft tissue lesion in the ankle and lower one third of right leg encasing the tendons, distal tibia and fibula and invading the muscles, skin and subcutaneous tissue with cortical erosions and marrow edema in the distal third of tibia and fibula

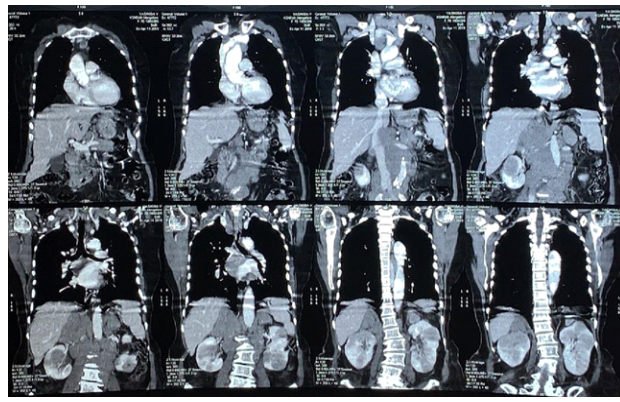

Figure 4 : CECT abdomen and pelvis revealed multiple retroperitoneal enlarged lymph nodes with multiple metastases to adrenal and kidneys. 


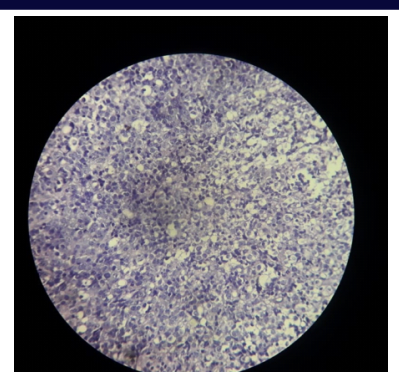

Figure 5: Sections from distal tibial biopsy shows necrotic and sclerotic bony bits with admixed atypical lymphoid cells with mildly pleomorphic nucleus, condensed chromatin and scanty cytoplasm.

\section{Competing Interests:}

The authors declare that they have no competing interests.

\section{Acknowledgements and Funding:}

Nil

\section{REFERENCES}

. Dubey P, Ha CS, Besa PC, et al. Localized primary malignant lymphoma of bone. Int J Radiat Oncol Biol Phys 1997; 37:1087.

2. Jawad MU, Schneiderbauer MM, Min ES, et al. Primary lymphoma of bone in adult patients. Cancer 2010; 116:871.

3. Mulligan ME, McRae GA, Murphey MD. Imaging features of primary lymphoma of bone. AJR Am J Roentgenol 1999; 173:1691.

4. Cai L, Stauder MC, Zhang YJ, et al. Early-stage primary bone lymphoma: a retrospective, multicenter Rare Cancer Network (RCN) Study. Int J Radiat Oncol Biol Phys 2012; 83:284.

5. Bruno Ventre M, Ferreri AJ, Gospodarowicz M, et al. Clinical features, management, and prognosis of an international series of 161 patients with limited-stage diffuse large B-cell lymphoma of the bone (the IELSG-14 study). Oncologist 2014; 19:291.

6. Messina C, Ferreri AJ, Govi S, et al. Clinical features, management and prognosis of multifocal primary bone lymphoma: a retrospective study of the international extranodal lymphoma study group (the IELSG 14 study). Br J Haematol 2014; 164:834.

7. Catlett JP, Williams SA, O'Connor SC, et al. Primary lymphoma of bone: an institutional experience. Leuk Lymphoma 2008; 49:2125.

8. Power DG, McVey GP, Korpanty G, et al. Primary bone lymphoma: single institution Power DG, McVey GP, Korpanty G,

9. Ford DR, Wilson D, Sothi S, et al. Primary bone lymphoma--treatment and outcome. Clin Oncol (R Coll Radiol) 2007; 19:50.

10. Alencar A, Pitcher D, Byrne G, Lossos IS. Primary bone lymphoma--the University of Miami experience. Leuk Lymphoma 2010; 51:39. 